\title{
KDM6A mediated expression of the long noncoding RNA DINO causes TP53 tumor suppressor stabilization in Human Papillomavirus type 16 E7 expressing cells
}

\author{
Surendra Sharma ${ }^{1,2}$, Karl Munger ${ }^{1,2 *}$
}

${ }^{1}$ Biochemistry Program, Graduate School of Biomedical Sciences, Tufts University School of Medicine, Boston MA 02111;

${ }^{2}$ Department of Developmental, Molecular and Chemical Biology, Tufts University School of Medicine, Boston MA, 02111.

Running title: TP53 stabilization by HPV16 E7 through DINO

* to whom correspondence should be addressed to at

Department of Developmental, Molecular and Chemical Biology, Tufts University School of Medicine, 136 Harrison Ave., MV701G

Boston, MA 02111, USA

Phone: 617-636-0306

Fax: 617-636-2990

Email: karl.munger@tufts.edu

Word counts: Abstract (226); Importance (75); Text without figure legends and references $(3,565)$ 


\section{ABSTRACT}

2 HPV16 E7 has long been noted to stabilize the TP53 tumor suppressor. However, the molecular

3 mechanism of TP53 stabilization by HPV16 E7 has remained obscure and can occur independent

4 of E2F regulated MDM2 inhibitor, p14 ${ }^{\mathrm{ARF}}$. Here, we report that the Damage Induced Noncoding

5 (DINO) lncRNA (DINOL) is the missing link between HPV16 E7 and increased TP53 levels.

6 DINO levels are decreased in cells where TP53 is inactivated, either by HPV16 E6, expression

7 of a dominant negative TP53 minigene or by TP53 depletion. DINO levels are increased in

8 HPV16 E7 expressing cells. HPV16 E7 causes increased DINO expression independent of RB1

9 degradation and E2F1 activation. Similar to the adjacent CDKN1A locus, DINO expression is

10 regulated by the histone demethylase, KDM6A. DINO stabilizes TP53 in HPV16 E7 expressing

11 cells and as a TP53 transcriptional target, DINO levels further increase. Similar to other

12 oncogenes such as adenovirus E1A or MYC, HPV16 E7 expressing cells are sensitized to cell

13 death under conditions of metabolic stress and in the case of E7, this has been linked to TP53

14 activation. Consistent with earlier studies, we show that HPV16 E7 expressing keratinocytes are

15 highly sensitive to metabolic stress induced by the antidiabetic drug, metformin. Metformin

16 sensitivity of HPV16 E7 expressing cells is rescued by DINO depletion. This work identifies

17 DINO as a critical mediator TP53 stabilization and activation in HPV16 E7 expressing cells. 


\section{IMPORTANCE}

20 Viral oncoproteins, including HPV16 E6 and E7 have been instrumental in elucidating the

21 activities of cellular signaling networks including those governed by the TP53 tumor suppressor.

22 Our study demonstrates that the long noncoding RNA DINO is the long sought missing link

23 between HPV16 E7 and elevated TP53 levels. Importantly, the TP53 stabilizing DINO plays a

24 critical role in the predisposition of HPV16 E7 expressing cells to cell death under metabolic

25 stress conditions from metformin treatment.

\section{KEYWORDS}

28 Human papillomavirus, E7, lncRNA, DINO, DINOL, TP53, metformin, metabolism, KDM6A 
INTRODUCTION

31 The cancer-associated, high-risk human papillomavirus (HPV) E6 and E7 proteins have

32 oncogenic activities and are necessary for tumor induction and maintenance. These low-

33 molecular weight proteins lack intrinsic enzymatic activities and function by subverting host

34 cellular regulatory networks $(1,2)$. Over the years, many potential cellular targets of the HPV E6

35 and E7 proteins have been identified. The main focus of these studies, however, has been on

36 identifying host cellular protein targets and the resulting dysregulation of protein coding

37 mRNAs. Protein coding genes, however, only constitute a minor fraction $(<3 \%)$ of the human

38 transcriptome (3), and advances in next generation sequencing technologies have shown that

39 noncoding RNAs including microRNAs and long noncoding (lnc) RNAs, are also aberrantly

40 expressed in HPV E6 and/or E7 expressing cells (4-7). It is now firmly established that

41 noncoding RNAs are important regulators of a variety of cellular processes that drive specific

42 biological phenotypes (8-11).

44 Many viral proteins, including the E6 and E7 proteins encoded by high-risk HPVs have evolved

45 to modulate the TP53 tumor suppressor pathway (12). TP53 is critically involved in sensing

46 many forms of cellular stress, including those caused by viral infection or nutrient starvation.

47 Upon activation, TP53 is stabilized and elicits transcriptional programs that trigger cytostatic or

48 cytotoxic responses, including cycle arrest, senescence or apoptosis (13). Hence, TP53 levels and

49 activity are tightly regulated. A well-known regulator of TP53 is the MDM2 ubiquitin ligase.

50 MDM2 is a TP53 responsive gene, which in turns binds and targets TP53 for ubiquitin dependent

51 proteasomal degradation (14). MDM2 activity is regulated at multiple levels. One important 
52 inhibitor of MDM2 is the p14 ${ }^{\mathrm{ARF}}$ protein, which is regulated by E2F transcription factors, the

53 downstream effectors of the retinoblastoma (RB1) tumor suppressor.

55 The E6 and E7 proteins encoded by high-risk HPVs, including HPV16, each modulate TP53

56 levels and activity. HPV16 E6 targets TP53 for proteasome mediated degradation through an

57 MDM2 independent mechanism that involves the E6-associated ubiquitin ligase, UBE3A

58 (E6AP) $(15,16)$. The ability of high-risk HPV E6 proteins to inhibit TP53 is thought to have

59 evolved from the necessity to counteract TP53 stabilization and activation triggered by

60 expression of the HPV E7 oncoprotein (17-22). Consequently, several other DNA tumor viruses,

61 including adenoviruses and many polyomaviruses that encode proteins that inactivate the RB1

62 tumor suppressor also encode proteins that dampen TP53 tumor suppressor activity. In the case

63 of the adenovirus E1A protein, TP53 stabilization was shown to be mediated by the E2F induced

64 MDM2 inhibitor, p14 ${ }^{\mathrm{ARF}}$ (23). Surprisingly, however, HPV16 E7 has been shown to stabilize

65 TP53 independently of p14 ${ }^{\mathrm{ARF}}(21)$ and, to this date, the mediator of HPV16 E7 mediated TP53

66 stabilization has remained elusive.

68 In recent years, it has been appreciated that various host cellular noncoding RNAs also function

69 as important regulators of the TP53 tumor suppressor pathway. In addition to microRNAs (24),

70 a variety of cellular lncRNAs have been identified as components of the TP53 signaling network

71 (25). LncRNAs are defined as transcripts longer than 200 base pairs with no or limited $(<100$

72 amino acids) protein coding capacity. LncRNAs are versatile molecules and can form complexes

73 with RNA, DNA and proteins. Some lncRNAs including lincRNA-p21, PURPL, MEG3 and

74 DINO are transcriptional targets of TP53, and can also modulate TP53 levels and activity (26- 
29). DINO (Damage Induced Noncoding) lncRNA expression is induced by TP53 as a consequence of DNA damage. It has been reported that DINO binds TP53, thereby stabilizing

77 and activating it. Hence, DINO functionally counteracts MDM2. In the present study,

78 we report that DINO is expressed at high levels in HPV16 E7 expressing cells, and this

79 upregulation correlates with the ability of HPV16 E7 to cause TP53 stabilization. High level

80 DINO expression in HPV16 E7 expressing cells is driven by the KDM6A histone demethylase

81 and TP53, and DINO, in turn, is necessary for maintaining elevated TP53 levels in HPV16 E7

82 expressing cells. Moreover, we show that the sensitivity of cells to undergo cell death in

83 response to metabolic stress can be controlled by modulating DINO levels.

RESULTS

\section{DINO levels correlate with TP53 levels in HPV oncoprotein expressing cells}

89 Expression of high-risk HPV E6 and E7 proteins is known to modulate TP53 levels and activity.

90 While HPV16 E7 expression causes TP53 stabilization, HPV16 E6 expression causes TP53

91 degradation. Hence, we determined whether the levels of DINO, a TP53 driven lncRNA

92 correlate with TP53 levels in HPV16 oncogene expressing primary Human Foreskin

93 Keratinocytes (HFKs). HFKs with expression of a dominant negative (dn)TP53 C-terminal

94 fragment were used as a control (30). We assessed TP53 levels by immunoblotting and

95 quantified DINO levels by quantitative reverse transcription polymerase chain reaction (qRT-

96 PCR). Compared to control HFKs, we detected lower DINO levels in HFK populations where

97 TP53 is inactivated either by the expression of the HPV16 E6 protein or by the dnTP53 C- 
98 terminal minigene (E6: $0.14+/-0.01, \mathrm{p}<0.001$, E6/E7: $0.22+/-0.04, \mathrm{p}<0.001$ and dnTP53:

$990.28+/-0.01, \mathrm{p}<0.001)$ (Figure 1B). This is consistent with a previous study that showed that

100 DINO expression is controlled by TP53 (28). In contrast, HPV16 E7 expressing HFKs, which, as

101 published previously $(17,20,21)$, express high levels of TP53 (Figure 1A), expressed

102 significantly increased DINO levels (E7: 6.14 +/- 0.14, p < 0.001) (Figure 1B). Hence, DINO

103 levels correlate with TP53 levels in HPV oncoprotein expressing cells.

104

\section{TP53 driven DINO causes elevated TP53 levels in HPV16 E7 expressing cells}

106 Given the correlation between DINO and TP53 levels in E7 expression cells, we next determined

107 whether DINO expression in HPV16 E7 expressing cells was driven by TP53. We transiently

108 depleted TP53 in control as well as in HPV16 E7 expressing human telomerase immortalized

109 HFKs (iHFKs) with a pool of TP53 targeting siRNAs. To assess the effect of TP53 depletion on

110 expression of TP53 target genes, we analyzed expression of CDKN1A (p21 $\left.{ }^{\mathrm{CIP} 1}\right)$ by western

111 blotting and qRT-PCR. As expected, CDKN1A protein and mRNA levels were decreased in

112 control and HPV16 E7 expressing cells upon TP53 depletion (Figure 2A, B). Similarly, TP53

113 depletion also caused a significant decrease of DINO in control as well as in HPV16 E7

114 expressing cells $(\mathrm{C}: 0.09+/-0.01, \mathrm{p}<0.001$ and E7: $0.08+/-0.02, \mathrm{p}<0.001)$ (Figure $2 \mathrm{~B}$ ).

115

116 To determine whether DINO may be the "missing link" that mediates TP53 stabilization in E7

117 expressing cells, we acutely depleted DINO in HPV16 E7 expressing iHFKs using doxycycline

118 regulated shRNA expression. Depletion of DINO after doxycycline treatment for 48 hours by

119 two different shRNAs each caused an approximately 50\% decrease in TP53 levels in E7

120 expressing cells (Figure 2C). Similarly, mRNA levels of the TP53 transcriptional target gene, 
121 CDKN1A gene were also decreased (sh-DINO1: $0.61+/-0.01, \mathrm{p}<0.01$, and sh-DINO2: $0.48+/-$

$1220.02, \mathrm{p}<0.01)$ (Figure 2D). Hence, DINO levels accumulate in HPV16 E7 expressing cells as a

123 result of TP53 activation, and, in addition, they amplify TP53 stabilization and presumably TP53

124 activation in HPV16 E7 expressing cells.

126 TP53 and KDM6A, but not RB1 and E2F1 are the upstream regulators of DINO

127 Previous mutational studies with HPV16 E7 revealed that TP53 stabilization by HPV16 E7

128 required similar sequences as those that are necessary for RB1 destabilization (20). Consistent

129 with these earlier studies, the RB1 binding and degradation defective HPV16 E7 $\triangle \mathrm{DLYC}$ mutant

130 failed to stabilize TP53 (Figure 3A). Similarly, unlike wild type HPV16 E7, expression of

131 HPV16 E7 $\triangle$ DLYC also failed to upregulate DINO in IMR90s normal human diploid lung

132 fibroblasts (E7: $7.03+/-1.28, \mathrm{p}<0.01$ and $\triangle \mathrm{DLYC}: 1.00+/-0.18$, non-significant) (Figure 3B).

133 To determine whether DINO levels in HPV16 E7 expressing cells were increased as a

134 consequence of RB1 tumor suppressor degradation and E2F activation, we transiently depleted

135 RB1 and E2F1 in control and HPV16 E7 expressing iHFKs by transfecting the corresponding

136 siRNA pools. Transfections of a TP53 targeting siRNA pool and a non-targeting siRNA pool

137 were used as positive and negative controls, respectively. Depletion of the corresponding

138 proteins was assessed by western blotting (Figure 3C). Whereas, as expected, TP53 depletion

139 caused a significant decrease of DINO levels, RB1 and E2F1 depletion did not cause significant

140 changes of DINO levels in control ( $\mathrm{siC}: 1.00+/-0.12$; siRB1: $1.15+/-0.03, \mathrm{p}=$ non-significant

141 (ns) and siE2F1: $0.96+/-0.14, \mathrm{p}=\mathrm{ns})$ or HPV16 E7 expressing cells (siC: 6.64 +/- 0.52, siRB1:

$1425.58+/-0.56, \mathrm{p}=\mathrm{ns}$ and siE2F1: $6.15+/-0.04, \mathrm{p}=\mathrm{ns}$ ) (Figure 3D). The DINO locus is in close

143 proximity of the CDKN1A locus (28), which is subject to epigenetic de-repression by the histone 
144 H3 lysine 27 (H3K27) demethylase, KDM6A $(31,32)$. Hence, we next tested whether DINO

145 expression is also regulated by KDM6A. Consistent with previous reports $(32,33)$, we found that

146 KDM6A expression was increased in E7 expressing cells. Moreover, DINO expression was

147 significantly decreased upon KDM6A depletion (C: $0.61+/-0.09, \mathrm{p}<0.01$ and E7: $2.83+/-0.17$,

$148 \mathrm{p}<0.001)($ Figure 3D). Hence, HPV16 E7 causes increased DINO expression through a

149 mechanism that involves KDM6A mediated de-repression.

151 Heightened metformin sensitivity of HPV16 E7 expressing cells is reversed upon depletion

152 of DINO

153 Our research group has previously reported that HPV16 E7 expressing fibroblasts are sensitized

154 to undergo cell death under conditions of serum starvation. This response was shown to be TP53

155 dependent since it was abrogated by co-expression of the HPV16 E6 protein or a dominant

156 negative TP53 minigene $(18,20)$. Given our results that DINO is critical to TP53 stabilization

157 and activation in HPV16 E7 expressing cells, we next wanted to determine whether vulnerability

158 of HPV16 E7 expressing cells could be controlled by modulating DINO levels. Since

159 keratinocytes are grown in serum free media, we first needed to define conditions that may

160 mimic growth factor deprivation in keratinocytes. We evaluated metformin, a drug that induces

161 metabolic stress by activating AMPK, inhibiting mTOR and mitochondrial respiratory complex

162 I, and depleting glycolytic and TCA cycle intermediates (34-37). Our results show that HPV16

163 E7 expressing HFKs are more vulnerable than control HFKs when subjected to metformin

164 treatment (C: 24.78+/- 1.12 and E7: 68.48 +/- 0.38, $\mathrm{p}<0.001)$ (Figure 4A). Heightened

165 metformin vulnerability of HPV16 E7 expressing cells was abrogated upon HPV16 E6 co-

166 expression $(-0.46+/-0.20, \mathrm{p}<0.001)$, consistent with the model that the vulnerability to 
167 metformin may be a consequence of E7 mediated TP53 stabilization and activation. To

168 determine whether DINO may modulate metformin induced cell death, we depleted DINO in

169 HPV16 E7 expressing primary HFKs and treated them with metformin. As expected, DINO

170 depletion caused reduced levels of TP53 and decreased expression of the TP53 transcriptional

171 target CDKN1A (Figure 4C, D). DINO depletion did not affect the viability of HPV16 E7

172 expressing HFKs when grown under standard tissue culture conditions. In contrast, however,

173 DINO depleted HPV16 E7 expressing HFKs were significantly more resistant (sh-control: 50.76

$174+/-0.78$, sh-DINO1: $-1.71+/-0.47, \mathrm{p}<0.001$ and sh-DINO2: $-10.21+/-0.68, \mathrm{p}<0.001)$ to

175 metformin treatment than normal HPV16 E7 expressing HFKs (Figure 4B). Hence, metformin

176 can be utilized to investigate the metabolic vulnerability of HPV16 E7 expressing cells and

177 DINO regulates cellular response to metformin induced metabolic stress.

\section{DISCUSSION}

181 The TP53 tumor suppressor is a central hub that integrates various cellular stress signals and

182 triggers appropriate cytostatic and cytotoxic responses. In normal cells, TP53 has a very short

183 half-life and is present at low levels because the TP53 regulated MDM2 ubiquitin ligase targets

184 TP53 for proteasomal degradation. Oncogenic insults can trigger TP53 activation. In response to

$185 \mathrm{RB} 1$ tumor suppressor inactivation, the E2F regulated $\mathrm{p} 14^{\mathrm{ARF}}$ protein inhibits MDM2 and causes

186 TP53 activation. However, it became clear early on that there must be additional regulators of

187 TP53, since p19ARF null mouse embryo fibroblasts were shown to still be capable of TP53

188 stabilization and activation in response to DNA damage (38). Similarly, even though HPV16 E7

189 causes E2F activation, it can stabilize and activate TP53 in p19 ${ }^{\mathrm{ARF}}$ null mouse embryo fibroblasts 
and the mechanism of HPV16 E7-mediated TP53 stabilization and activation has remained

191 elusive (21).

192

193 DINO is a TP53 responsive gene and DINO levels increase in response to DNA damage. DINO

194 was reported to bind and stabilize TP53, thereby amplifying the TP53 transcriptional response to

195 DNA damage. Hence, DINO functionally counteracts MDM2 (28, 39). Here, we show that

196 DINO expression correlates with TP53 expression in HPV16 oncoprotein expressing cells and is

197 highly expressed in HPV16 E7 expressing keratinocytes. DINO depletion causes a decrease in

198 TP53 levels in HPV16 E7 expressing cells and, thus, DINO is the missing link between HPV16

199 E7 expression and TP53 stabilization. The RB1 binding/degradation defective HPV16 E7

$200 \Delta$ DLYC mutant, which was previously shown to be defective for TP53 stabilization (20), does

201 not cause increased DINO expression. Hence, we were quite surprised that we did not detect any

202 changes in DINO expression when RB1 was depleted by RNAi. This suggests that RB1

203 degradation and the ensuing activation of E2F are not the primary triggers of DINO expression.

204 In contrast, however, depletion of the H3K27 demethylase KDM6A, which is expressed at

205 higher levels in E7 expressing cells, causes a significant decrease in DINO. Hence our results,

206 suggest a model whereby E7 mediated upregulation of KDM6A provides the initial trigger for

207 DINO induction. Our current results cannot distinguish, however, whether, similar to the

208 proximal CDKN1A locus $(28,32)$, DINO expression is directly de-repressed through KDM6A

209 mediated H3K27 demethylation, or whether this involves an indirect mechanism. It is noted,

210 however, that DINO and CDKN1A are distinct genetic loci and are expressed from opposite

211 strands in divergent directions (28). According to our model, the initial KDM6A mediated

212 increase in DINO expression causes TP53 stabilization and activation, which in turn causes a 
213 further increase in DINO and TP53. Since E7 will also cause increased expression of p14 ${ }^{\mathrm{ARF}}$ as a

214 consequence of RB1 degradation and E2F activation, this may impair MDM2 activity and cause

215 a further increase in TP53 and DINO (Figure 5). Importantly, this model, where the initial

216 trigger of DINO expression is through KDM6A, accommodates the finding E7 expression can

217 cause increased TP53 levels in p19 ARF deficient mouse embryo fibroblasts (21).

219 Having established DINO as the key mediator of elevated TP53 levels in E7 expressing cells, we 220 next wanted to determine whether DINO may not only modulate TP53 levels but also a known 221 TP53 dependent biological phenotype of HPV16 E7 expressing cells. We previously discovered 222 that, similar to adenovirus E1A or MYC, HPV16 E7 expressing primary cells undergo cell death

223 when deprived of growth factors $(17,20,40-42)$. This form of cell death is triggered by

224 conflicting growth signals; the proliferative signal generated by oncogene expression that clashes

225 with the antiproliferative signal as a consequence of serum deprivation. This likely represents a

226 cell-intrinsic, innate tumor suppressive response that has been dubbed the "trophic sentinel

227 response" (43). In the case of HPV16 E7, this response is TP53 dependent and is overridden by 228 co-expression of the HPV16 E6 oncoprotein $(18,20)$.

230 Serum deprivation is not a practicable approach to induce metabolic stress in keratinocytes, the

231 normal host cells of HPVs, since they are grown in serum free media. Hence, we evaluated other

232 means of generating metabolic stress and used metformin, a member of anti-diabetic biguanide

233 compounds that are evaluated for repurposing as cancer therapeutic and/or chemoprevention

234 drugs. Metformin treatment of control and HPV16 E7 expressing keratinocytes revealed that, 
235 compared to parental cells, HPV16 E7 expressing cells are more sensitive to metformin

236 treatment and that DINO depletion abrogates this sensitivity.

238 Similar to microRNAs, lncRNA activity can be modulated by nucleic acid based inhibitors and

239 mimics and it will be interesting to determine whether DINO based compounds may be

240 developed that could be used to modulate the clinical response to drugs that cause metabolic

241 stress in cancer cells, particularly in cancer such as HPV associated cancer where TP53 is not

242 mutated.

245 MATERIALS AND METHODS:

247 Cell culture

248 Primary human foreskin keratinocytes (HFKs) were prepared from a pool of 3 to 5 neonatal

249 foreskins obtained anonymously from the Ob/Gyn department at Tufts Medical Center. Human

250 telomerase (hTERT)-immortalized HFKs (iHFKs) were a generous gift from Dr. Aloysius

251 Klingelhutz (44). HFKs and iHFKs were grown and maintained in keratinocyte-serum-free

252 media (KSFM) supplemented with human recombinant epidermal growth factor and bovine

253 pituitary extract (Invitrogen). IMR-90s normal human diploid lung fibroblasts were purchased

254 from ATCC and maintained in Dulbecco's Modified Eagle Medium (DMEM) supplemented with

255 10\% fetal bovine serum. HPV 16 E6 and/or E7 and dominant negative (dn)TP53 C-terminal

256 minigene expressing cells were created by retroviral infection with recombinant LXSN based

257 retroviruses provided kindly by Dr. Denise Galloway (HPV oncogenes $(45,46))$ and Dr. Moshe 
Oren (dnTP53 (30)). Cells were selected in $300 \mu \mathrm{g} / \mathrm{ml} \mathrm{G418} \mathrm{(Gibco)} \mathrm{and} \mathrm{G418-resistant} \mathrm{pooled}$ cell populations were used for analysis. In all HFKs studies, donor and passage matched HFKs

260 of passages less than 8 times were used. In IMR90s studies, passage matched IMR90s of

261 passages less than 10 times were used. Doxycycline and polybrene were purchased from Sigma.

262 Metformin was purchased from Cayman Chemicals. Doxycycline and metformin were dissolved

263 in PBS and freshly prepared for each use.

\section{Western blotting and antibodies}

266 Lysates were prepared by incubating the cells in RIPA lysis buffer supplemented with Pierce

267 protease inhibitor (Thermo Scientific) and Pierce phosphatase inhibitor (Thermo Scientific) at 4

$268 \mathrm{C}$ for $30 \mathrm{~min}$. Proteins extracts were cleared by centrifugation at 15,000 g for $15 \mathrm{~min}$. Equal

269 amounts $(50 \mu \mathrm{g})$ of proteins were loaded and fractionated on 4-12\% NuPAGE Bis-Tris Gels

270 (Invitrogen). Protein were transferred to PVDF membranes (Millipore) and blocked with TNET

271 buffer (200 mM Tris-HCl, $1 \mathrm{M} \mathrm{NaCl}, 50 \mathrm{mM}$ EDTA, with 0.1\% Tween-20, pH 7.5) containing

$2725 \%$ nonfat dry milk at room temperature for 1 hour. Blots were incubated with following primary

273 antibodies at 4 C overnight: TP53 (OP43, Calbiochem, 1:1000), RB1 (Ab-5, Millipore, 1:100),

274 E2F1 (sc-251, Santa Cruz, 1:500), KDM6A (ab36938, Abcam, 1:300), CDKN1A (ab109520,

275 Abcam, 1:1,000), $\alpha$-tubulin (ab18251, Abcam, 1:1000) and actin (Ab-1501, Millipore, 1:1000).

276 Membranes were rinsed in TNET and incubated with the corresponding secondary antibodies,

277 horseradish peroxidase (HRP)-conjugated anti-mouse antibody (NA931, GE Healthcare Life

278 Sciences, 1:10,000) or HRP-conjugated anti-rabbit antibody (NA934V, GE Healthcare Life

279 Sciences, 1:10,000) for one hour at room temperature. Antigen/antibody complexes were

280 visualized by Enhanced Chemiluminescence (Life Technologies) and signals were digitally 
281 acquired on a G:Box Chemi-XX6 imager with GeneSys software (Syngene). Protein bands were 282 quantified using GeneTools Software (Syngene).

\section{Lentiviral expression plasmids}

285 Lentiviral vectors encoding two separate DINO targeting shRNA hairpin sequences were kind

286 gifts from Dr. Howard Chang (28). Doxycycline-inducible DINO knockdown vectors were

287 created by inserting these DINO targeting hairpin sequences into the Tet-pLKO-puro vector

288 backbone (Addgene plasmid \#21915, (47)). Oligonucleotide sequences used for cloning were,

289 sh-DINO1 oligo (5'-3'): ccggcacagaagaattggacattgaactcgagttcaatgtccaattcttctgtgttttt, and sh-

290 DINO2 oligo (5'-3'): ccggctggtttatggagatgacataactcgagttatgtcatctccataaaccagttttt. As a negative

291 control, non-mammalian shRNA sequences (Sigma SHC002) were cloned into Tet-pLKO-puro

292 vectors by using oligo (5'-3'): ccggcaacaagatgaagagcaccaactcgagttggtgctcttcatcttgttgttttt. All

293 inserted sequences were verified by DNA sequencing.

\section{RNA interference and lentiviral transduction}

$2963 \times 10^{6}$ control and HPV16 E7 iHFKs were seeded on $10 \mathrm{~cm}$ cell culture dish and allowed to

297 adhere overnight. Next day, cells were transfected with $30 \mathrm{nM}$ siRNAs specific for targeted 298 genes (ON-TARGETplus SMARTpools; Thermo Scientific Dharmacon) or a negative control 299 siRNA (Non-Targeting Pool; Thermo Scientific Dharmacon) using Lipofectamine RNAiMax

300 reagent (Invitrogen) per the manufacturer's instructions. Dharmacon references for gene specific

301 siRNAs used in this study are, TP53 (L-003329-00), RB1 (L-003296-02), E2F1 (L-003259-00),

302 KDM6A (L-014140-01) and non-targeting control (D-001810-10). Recombinant lentiviruses

303 expressing either non-targeting control or DINO shRNA sequences were made by transfecting 
304 HEK293T cells with the corresponding lentiviral vector, psPAX2 packaging (Addgene\#12260) and pMD2.G (Addgene\#12259) envelope plasmid DNA at a ratio of 4:3:1, respectively. Culture medium was collected at 48 hours post transfection and used for infection in conjunction with $0.4 \mathrm{ug} / \mathrm{mL}$ polybrene. Post 4 hours of infections, the inoculum was removed and replaced with fresh media. Stable cell populations were generated post selection in $1 \mu \mathrm{g} / \mathrm{ml}$ puromycin.

\section{RNA isolation and quantitative PCR}

311 Total RNA was isolated using the Quick-RNA MiniPrep (Zymo Research) and $1 \mu \mathrm{g}$ of total

312 RNA was reverse transcribed using the Quantitect Reverse Transcription Kit (Qiagen) per the

313 manufacturer's instructions. Quantitative PCR (qPCR) was performed in triplicate using SYBR

314 Green PCR Master Mix (Applied Biosystems) reagents in a StepOne Plus (Applied Biosystems)

315 thermocycler system. For all qPCR reactions in this study, thermocycler settings of $20 \mathrm{sec}$ at

$31695^{\circ} \mathrm{C}$, followed by 40 cycles of $3 \mathrm{sec}$ at $95^{\circ} \mathrm{C}$ and $30 \mathrm{sec}$ at $60^{\circ} \mathrm{C}$ were used. The following

317 qPCR primer sequences were used in this study. DINO: 5'-ggaggcaaaagtcctgtgtt -3'(forward)

318 and 5'- gggctcagagaagtctggtg -3'(reverse); CDKN1 A: 5'- catgtggacctgtcactgtcttgta -3'(forward)

319 and 5' - gaagatcagccggcgtttg -3'(reverse); RPLP0:5'- atcaacgggtacaaacgagtc -3'(forward) and 5'-

320 cagatggatcagccaagaagg -3 '(reverse). The expression data shown was quantified using the $\Delta \Delta \mathrm{CT}$

321 method by normalizing all the qPCR targets against a housekeeping gene, RPLP0.

\section{Cell viability}

324 Cell viability was assessed using a resazurin assay, as previously described (48). At the day of 325 cell viability reading, cells were incubated with fresh media containing $10 \mu \mathrm{g} / \mathrm{ml}$ resazurin 
326 (Sigma). After a one-hour incubation, fluorescence readings were recorded on a Synergy H1

327 microplate reader (BioTek) using $560 \mathrm{~nm}$ excitation and $590 \mathrm{~nm}$ emission filters.

\section{ACKNOWLEDGMENTS}

332 We thank Dr. Howard Chang (Stanford University) for generously sharing DINO reagents, Dr.

333 Al Klingelhutz (University of Iowa) for providing telomerase immortalized human foreskin 334 keratinocytes and Drs. Amy Yee, Philip Hinds, Peter Juo, Claire Moore and members of the

335 Munger Lab for stimulating discussions and valuable suggestions throughout the course of this

336 work. Supported by PHS grants AI147176, CA066980 and CA228543 (K.M.) and a Dean's

337 Fellowship and a Tufts Collaborative Cancer Biology Award from the Tufts Graduate School of 338 Biomedical Sciences (S.S.).

341 FIGURE LEGENDS:

343 Figure 1: DINO levels corelate with TP53 levels in HPV oncoprotein expressing cells. HFK

344 populations with stable expression of HPV16 E6 and/or E7 or a dominant negative TP53

345 minigene (dn TP53) were validated by determining the levels of RB1, TP53 and E7 by western

346 blotting (A). DINO levels were accessed in these HFK populations by qRT-PCR assays (B).

347 Levels shown are relative to control vector transduced HFKs. The bar graph depicts means \pm 
348 SEM $(\mathrm{n}=3)$ calculated from a single representative experiment. *** $\mathrm{p}<0.001$ (Student's t-test).

349 Similar results were obtained in three independently derived HFK populations.

351 Figure 2: DINO causes elevated TP53 levels in HPV16 E7 expressing cells.

352 TP53 depletion in both control (C) and HPV16 E7 (E7) expressing iHFKs were validated by

353 western blotting and assessment of the canonical TP53 transcriptional target CKDN1A protein

354 (A) and mRNA levels (B). DINO levels were determined in TP53 depleted iHFKs by qRT-PCR

355 assays and results are shown by comparing it with control siRNA transfected iHFKs. For acute

356 depletion of DINO, HPV16 E7 expressing iHFKs harboring doxycycline inducible DINO

357 shRNA expression vectors were treated with $1 \mu \mathrm{g} / \mathrm{mL}$ doxycycline for 48 hours. HPV16 E7

358 iHFKs with acute expression of non-targeting shRNA sequences were used as negative controls.

359 Levels of TP53 protein were determined by western blotting (C). Expression of the TP53

360 responsive CDKN1A mRNA and DINO depletion were assessed by qRT-PCR (D). Bar graphs

361 depict means $\pm \operatorname{SEM}(\mathrm{n}=3)$ calculated from a single representative experiment. $* * * \mathrm{p}<0.001, * *$

$362 \mathrm{p}<0.01, * \mathrm{p}<0.05$ (Student's t-test). Similar results were obtained in three independent

363 experiments.

Figure 3: KDM6A and TP53, but not RB1 and E2F1 are the upstream regulators of DINO.

366 IMR90 cell populations expressing either control, wild type HPV16 E7 or the RB1

367 binding/degradation defective HPV16 E7 $\triangle \mathrm{DLYC}$ mutant, were assessed for RB1 degradation,

368 TP53 stabilization and E2F1 expression by western blotting (A). DINO levels were accessed in

369 these IMR90s populations by qRT-PCR (B). Levels shown are relative to control vector

370 transduced IMR90s. Validation of TP53, RB1, E2F1 and KDM6A depletion in control (C) and 
371 HPV16 E7 (E7) expressing iHFKs by western blotting (C). DINO levels in each of these lines as

372 determined by qRT-PCR (D). Bar graphs represent means \pm SEM $(n=3)$ calculated from a single

373 representative experiment are shown. $* * * \mathrm{p}<0.001, * * \mathrm{p}<0.01$ and $\mathrm{ns}=$ non-significant

374 (Student's t-test). Similar results were obtained in three independent experiments.

375

376

Figure 4: Heightened metformin sensitivity of HPV16 E7 expressing cells is reversed upon

377 DINO depletion. Cell viability as assessed by the resazurin assay of control, HPV16 E7 and

378 HPV16 E6/E7 expressing HFKs that were either untreated (mock) or treated with $20 \mathrm{mM}$

379 metformin for 96 hours (A). Viability of HPV16 E7 expressing HFKs with expression of either

380 of two DINO specific shRNAs or a scrambled control shRNA (B). TP53 and CDKN1A

381 expression in these cell lines as determined by western blotting (C). Expression of CDKN1A

382 mRNA and validation of DINO depletion in these HFK populations as determined by qRT-PCR

383 (D). Bar graphs depict means \pm SEM $(n=3)$ calculated from a single representative experiment.

$384 * * * \mathrm{p}<0.001, * * \mathrm{p}<0.01, * \mathrm{p}<0.05, \# \mathrm{p}<0.001$ (Student's t-test). Similar results were

385 obtained in three independently derived HFK populations.

Figure 5: Model of TP53 stabilization and activation by the HPV16 E7 oncoprotein. HPV16

388 E7 expression triggers DINO expression through a KDM6A mediated pathway. This causes

389 TP53 activation which results in further TP53 mediated DINO transcription. HPV16 E7 also

390 induces $\mathrm{p} 14^{\mathrm{ARF}}$ as a consequence of RB1 degradation which inhibits MDM2 ubiquitin ligase

391 activity causing a further increase in TP53 and DINO. See text for details. 


\section{REFERENCES:}

395 1. Rozenblatt-Rosen O, Deo RC, Padi M, Adelmant G, Calderwood MA, Rolland T, 396 Grace M, Dricot A, Askenazi M, Tavares M, Pevzner SJ, Abderazzaq F, Byrdsong D, Carvunis AR, Chen AA, Cheng J, Correll M, Duarte M, Fan C, Feltkamp MC, Ficarro SB, Franchi R, Garg BK, Gulbahce N, Hao T, Holthaus AM, James R, Korkhin A, Litovchick L, Mar JC, Pak TR, Rabello S, Rubio R, Shen Y, Singh S,

2. Wu SC, Canarte V, Beeravolu H, Grace M, Sharma S, Munger K. 2020. Chapter 4 doi:https://doi.org/10.1016/B978-0-12-814457-2.00004-0. Academic Press. 
416 4. Gunasekharan V, Laimins LA. 2013. Human papillomaviruses modulate microRNA

417145 expression to directly control genome amplification. J Virol 87:6037-6043.

418 5. Harden ME, Prasad N, Griffiths A, Munger K. 2017. Modulation of microRNA-

419 mRNA Target Pairs by Human Papillomavirus 16 Oncoproteins. MBio 8.

$420 \quad$ 6. He H, Liu X, Liu Y, Zhang M, Lai Y, Hao Y, Wang Q, Shi D, Wang N, Luo XG, Ma

421 W, Zhang TC. 2019. Human Papillomavirus E6/E7 and Long Noncoding RNA

422 TMPOP2 Mutually Upregulated Gene Expression in Cervical Cancer Cells. J Virol 93.

$423 \quad$ 7. Sharma S, Munger K. 2018. Expression of the cervical carcinoma expressed PCNA

424 regulatory (CCEPR) long noncoding RNA is driven by the human papillomavirus E6

425 protein and modulates cell proliferation independent of PCNA. Virology 518:8-13.

426 8. Hutvagner G, Simard MJ, Mello CC, Zamore PD. 2004. Sequence-specific inhibition

427 of small RNA function. PLoS Biol 2:E98.

428 9. O'Brien J, Hayder H, Zayed Y, Peng C. 2018. Overview of MicroRNA Biogenesis, 429 Mechanisms of Actions, and Circulation. Front Endocrinol (Lausanne) 9:402.

430 10. Rinn JL, Chang HY. 2012. Genome regulation by long noncoding RNAs. Annu Rev $431 \quad$ Biochem 81:145-166.

432 11. Wilusz JE, Sunwoo H, Spector DL. 2009. Long noncoding RNAs: functional surprises 433 from the RNA world. Genes Dev 23:1494-1504.

434 12. Aloni-Grinstein R, Charni-Natan M, Solomon H, Rotter V. 2018. p53 and the Viral

435 Connection: Back into the Future (double dagger). Cancers (Basel) 10.

436 13. Lane DP. 1992. Cancer. p53, guardian of the genome. Nature 358:15-16. 
437 14. Momand J, Zambetti GP, Olson DC, George D, Levine AJ. 1992. The mdm-2 oncogene product forms a complex with the p53 protein and inhibits p53-mediated transactivation. Cell 69:1237-1245.

$440 \quad$ 15. Hengstermann A, Linares LK, Ciechanover A, Whitaker NJ, Scheffner M. 2001. Complete switch from Mdm2 to human papillomavirus E6-mediated degradation of p53 in cervical cancer cells. Proc Natl Acad Sci U S A 98:1218-1223.

443 16. Scheffner M, Huibregtse JM, Vierstra RD, Howley PM. 1993. The HPV-16 E6 and 75:495-505.

446 17. Demers GW, Halbert CL, Galloway DA. 1994. Elevated wild-type p53 protein levels in human epithelial cell lines immortalized by the human papillomavirus type $16 \mathrm{E} 7$ gene. Virology 198:169-174.

449 18. Eichten A, Rud DS, Grace M, Piboonniyom SO, Zacny V, Munger K. 2004. Molecular pathways executing the "trophic sentinel" response in HPV-16 E7-expressing

19. Eichten A, Westfall M, Pietenpol JA, Munger K. 2002. Stabilization and functional normal human diploid fibroblasts upon growth factor deprivation. Virology 319:81-93.

455 20. Jones DL, Thompson DA, Munger K. 1997. Destabilization of the RB tumor 456 suppressor protein and stabilization of p53 contribute to HPV type 16 E7-induced apoptosis. Virology 239:97-107. 
458 21. Seavey SE, Holubar M, Saucedo LJ, Perry ME. 1999. The E7 oncoprotein of human papillomavirus type 16 stabilizes p53 through a mechanism independent of p19(ARF). J Virol 73:7590-7598.

461 22. Zhou X, Munger K. 2009. Expression of the human papillomavirus type 16 E7 oncoprotein induces an autophagy-related process and sensitizes normal human keratinocytes to cell death in response to growth factor deprivation. Virology 385:192197.

23. de Stanchina E, McCurrach ME, Zindy F, Shieh SY, Ferbeyre G, Samuelson AV, Prives C, Roussel MF, Sherr CJ, Lowe SW. 1998. E1A signaling to p53 involves the p19(ARF) tumor suppressor. Genes Dev 12:2434-2442.

468 24. Feng Z, Zhang C, Wu R, Hu W. 2011. Tumor suppressor p53 meets microRNAs. Journal of molecular cell biology 3:44-50.

470 25. Chaudhary R, Lal A. 2017. Long noncoding RNAs in the p53 network. Wiley $471 \quad$ Interdiscip Rev RNA 8.

472 26. Huarte M, Guttman M, Feldser D, Garber M, Koziol MJ, Kenzelmann-Broz D, Khalil AM, Zuk O, Amit I, Rabani M, Attardi LD, Regev A, Lander ES, Jacks T, Rinn JL. 2010. A large intergenic noncoding RNA induced by p53 mediates global gene repression in the p53 response. Cell 142:409-419.

476 27. Li XL, Subramanian M, Jones MF, Chaudhary R, Singh DK, Zong X, Gryder B, 
Noncoding RNA PURPL Suppresses Basal p53 Levels and Promotes Tumorigenicity in Colorectal Cancer. Cell Rep 20:2408-2423.

482 28. Schmitt AM, Garcia JT, Hung T, Flynn RA, Shen Y, Qu K, Payumo AY, Peres-da-

Silva A, Broz DK, Baum R, Guo S, Chen JK, Attardi LD, Chang HY. 2016. An inducible long noncoding RNA amplifies DNA damage signaling. Nat Genet 48:13701376.

29. Zhou Y, Zhong Y, Wang Y, Zhang X, Batista DL, Gejman R, Ansell PJ, Zhao J,

30. Gottlieb E, Haffner R, von Ruden T, Wagner EF, Oren M. 1994. Down-regulation of wild-type p53 activity interferes with apoptosis of IL-3-dependent hematopoietic cells following IL-3 withdrawal. Embo j 13:1368-1374.

492 31. Kim D-H, Tang Z, Shimada M, Fierz B, Houck-Loomis B, Bar-Dagen M, Lee S, Lee S-K, Muir TW, Roeder RG, Lee JW. 2013. Histone H3K27 trimethylation inhibits H3 binding and function of SET1-like H3K4 methyltransferase complexes. Molecular and

32. Soto DR, Barton C, Munger K, McLaughlin-Drubin ME. 2017. KDM6A addiction of cervical carcinoma cell lines is triggered by E7 and mediated by p21CIP1 suppression of replication stress. PLoS Pathog 13:e1006661.

499 33. McLaughlin-Drubin ME, Crum CP, Münger K. 2011. Human papillomavirus E7 oncoprotein induces KDM6A and KDM6B histone demethylase expression and causes epigenetic reprogramming. Proceedings of the National Academy of Sciences of the United States of America 108:2130-2135. 
503 34. Hinke SA, Martens GA, Cai Y, Finsi J, Heimberg H, Pipeleers D, Van de Casteele M. 2007. Methyl succinate antagonises biguanide-induced AMPK-activation and death of pancreatic beta-cells through restoration of mitochondrial electron transfer. Br J Pharmacol 150:1031-1043.

$507 \quad$ 35. Kalender A, Selvaraj A, Kim SY, Gulati P, Brule S, Viollet B, Kemp BE, Bardeesy

N, Dennis P, Schlager JJ, Marette A, Kozma SC, Thomas G. 2010. Metformin, independent of AMPK, inhibits mTORC1 in a rag GTPase-dependent manner. Cell Metab 11:390-401.

Shaw RJ. 2009. LKB1 and AMP-activated protein kinase control of mTOR signalling

37. Stephenne X, Foretz M, Taleux N, van der Zon GC, Sokal E, Hue L, Viollet B, Guigas B. 2011. Metformin activates AMP-activated protein kinase in primary human

38. Kamijo T, van de Kamp E, Chong MJ, Zindy F, Diehl JA, Sherr CJ, McKinnon PJ. 1999. Loss of the ARF tumor suppressor reverses premature replicative arrest but not

39. Khanal T, Leung YK, Jiang W, Timchenko N, Ho SM, Kim K. 2019. NR2E3 is a key component in p53 activation by regulating a long noncoding RNA DINO in acute liver injuries. Faseb j 33:8335-8348.

522 40. Hermeking H, Eick D. 1994. Mediation of c-Myc-induced apoptosis by p53. Science 265:2091-2093.

524 41. Lowe SW, Ruley HE. 1993. Stabilization of the p53 tumor suppressor is induced by adenovirus 5 E1A and accompanies apoptosis. Genes Dev 7:535-545. 
526 42. Serrano M, Lin AW, McCurrach ME, Beach D, Lowe SW. 1997. Oncogenic ras Cell 88:593-602.

529 43. Evan GI, Vousden KH. 2001. Proliferation, cell cycle and apoptosis in cancer. Nature 411:342-348.

531 44. Farwell DG, Shera KA, Koop JI, Bonnet GA, Matthews CP, Reuther GW, Coltrera MD, McDougall JK, Klingelhutz AJ. 2000. Genetic and epigenetic changes in human epithelial cells immortalized by telomerase. The American journal of pathology

45. Demers GW, Espling E, Harry JB, Etscheid BG, Galloway DA. 1996. Abrogation of growth arrest signals by human papillomavirus type $16 \mathrm{E} 7$ is mediated by sequences required for transformation. J Virol 70:6862-6869.

538 46. Halbert CL, Demers GW, Galloway DA. 1991. The E7 gene of human papillomavirus type 16 is sufficient for immortalization of human epithelial cells. J Virol 65:473-478.

540 47. Wiederschain D, Wee S, Chen L, Loo A, Yang G, Huang A, Chen Y, Caponigro G, Yao Y-M, Lengauer C, Sellers WR, Benson JD. 2009. Single-vector inducible lentiviral RNAi system for oncology target validation. Cell cycle (Georgetown, Tex)

\section{48. Riss TL, Moravec RA, Niles AL, Duellman S, Benink HA, Worzella TJ, Minor L.}

545 2004. Cell Viability Assays. In Sittampalam GS, Grossman A, Brimacombe K, Arkin M, 
bioRxiv preprint doi: https://doi.org/10.1101/2020.01.01.892612; this version posted January 2, 2020. The copyright holder for this preprint (which was not certified by peer review) is the author/funder, who has granted bioRxiv a license to display the preprint in perpetuity. It is made available under aCC-BY-NC-ND 4.0 International license.

McManus O, Riss T, Saradjian P, Trask OJ, Jr., Weidner JR, Wildey MJ, Xia M, Xu X 
A iHFK

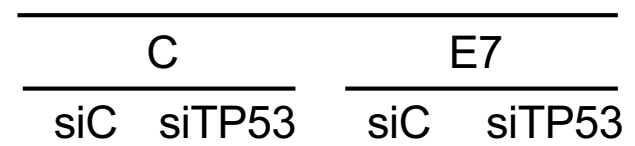

TP53

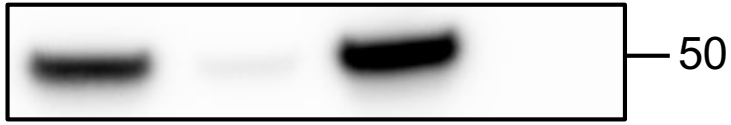

CDKN1A

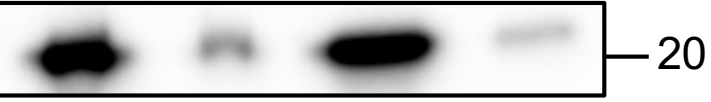

a-Tubulin

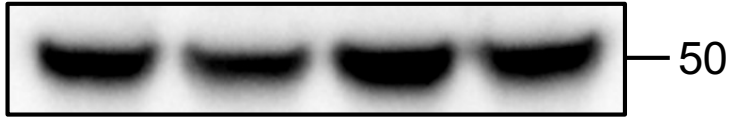

C

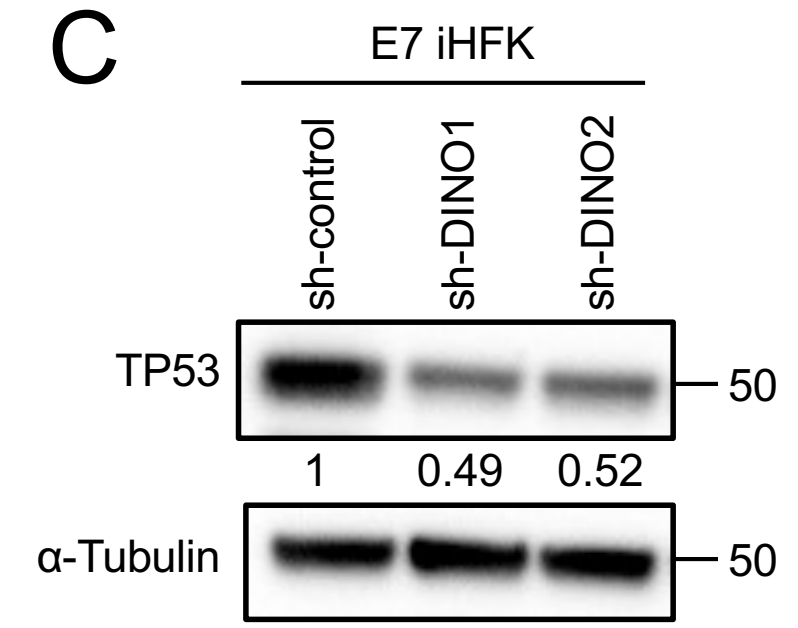

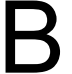

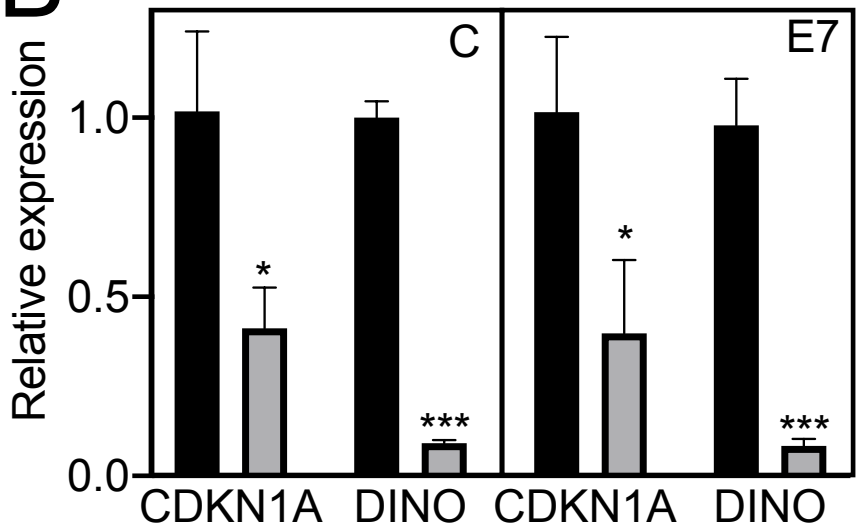

siC

$\square$ siTP53

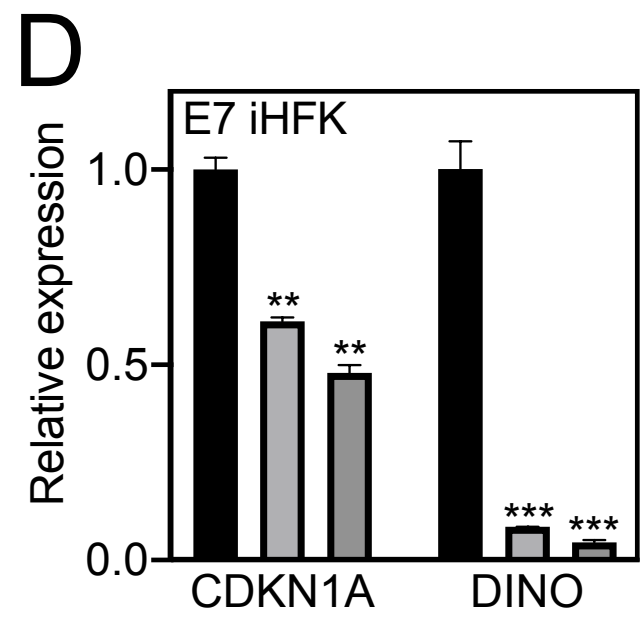

a sh-control

$\square$ sh-DINO1

$\square$ sh-DINO2 

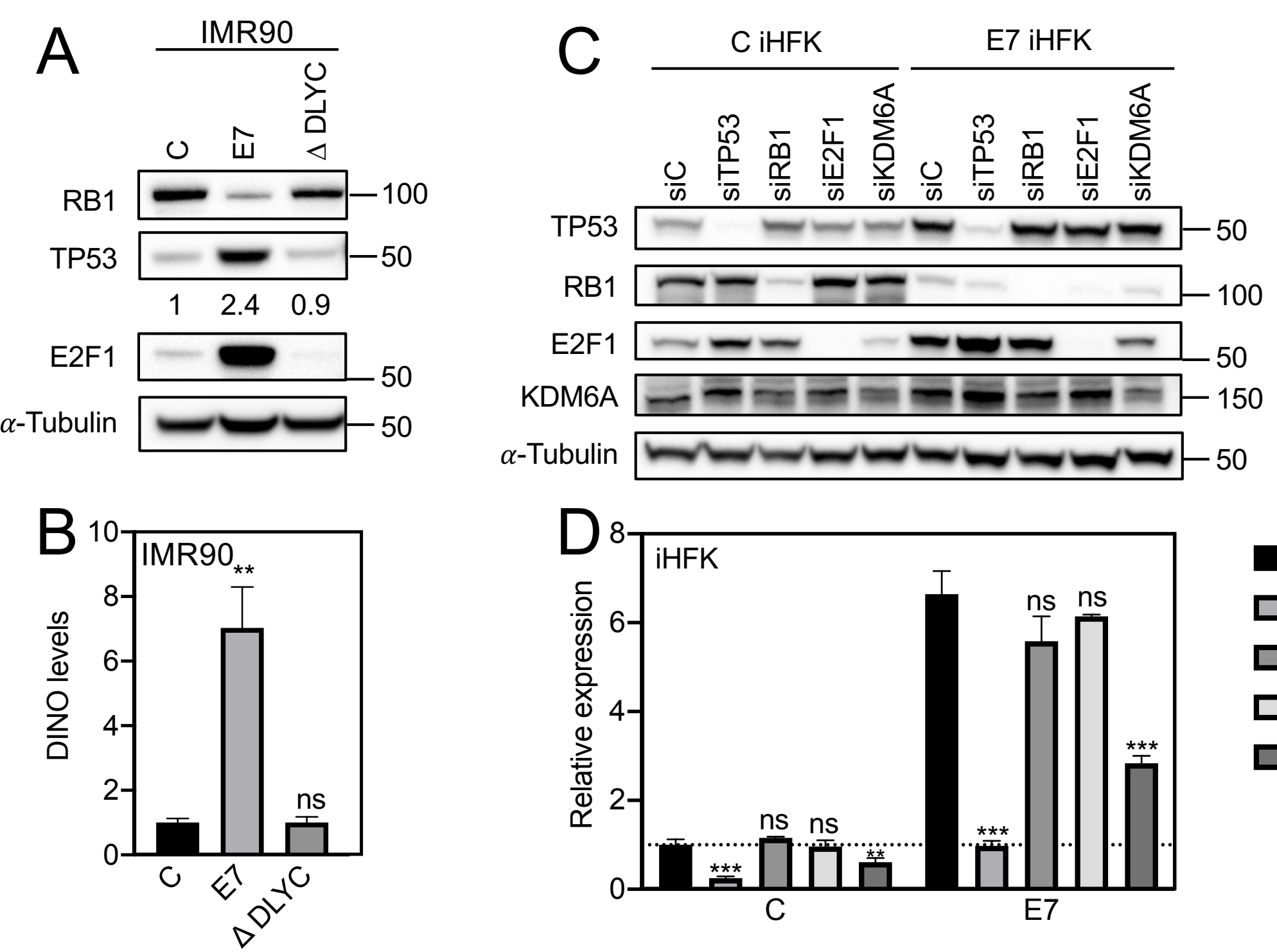

LiC

$\square$ siTP53

$\square$ siRB1

$\square$ siE2F1

$\square$ siKDM6A 


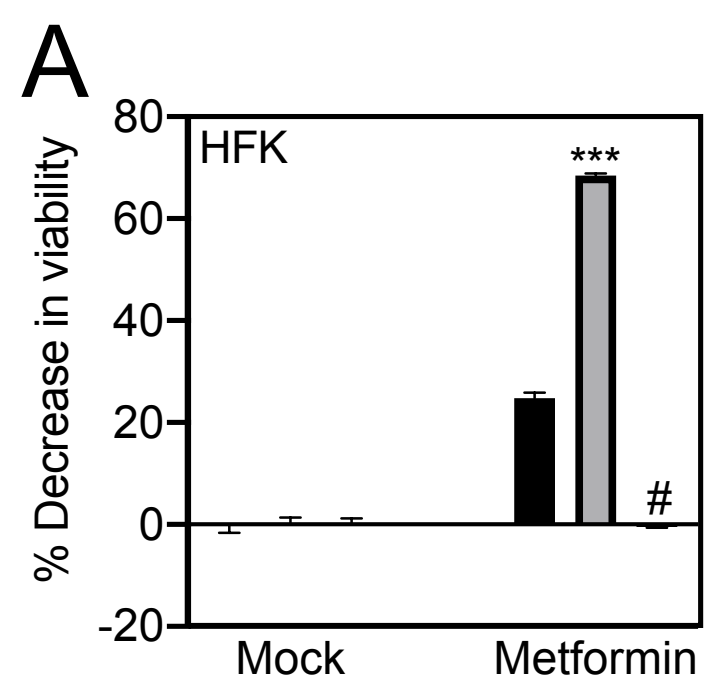

B

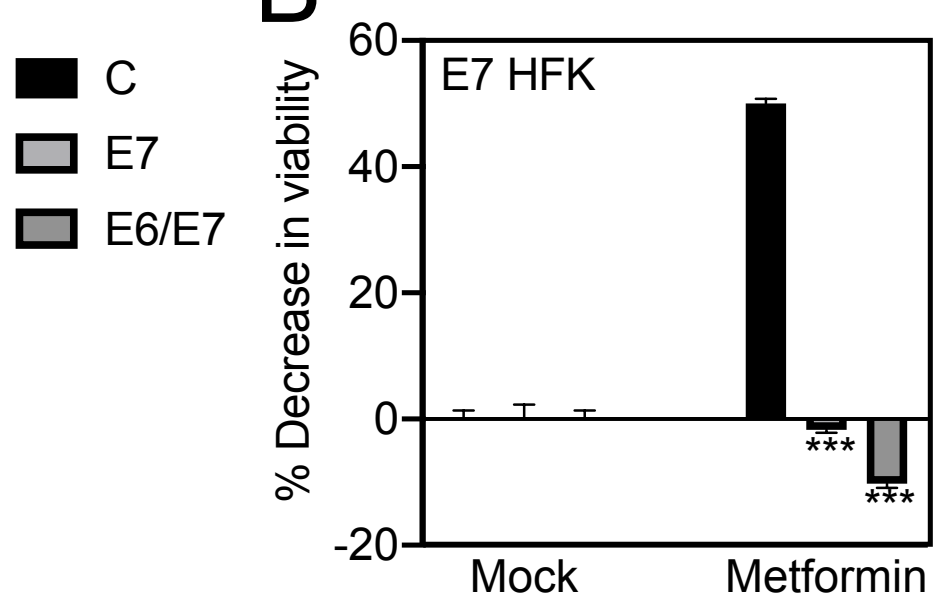

C
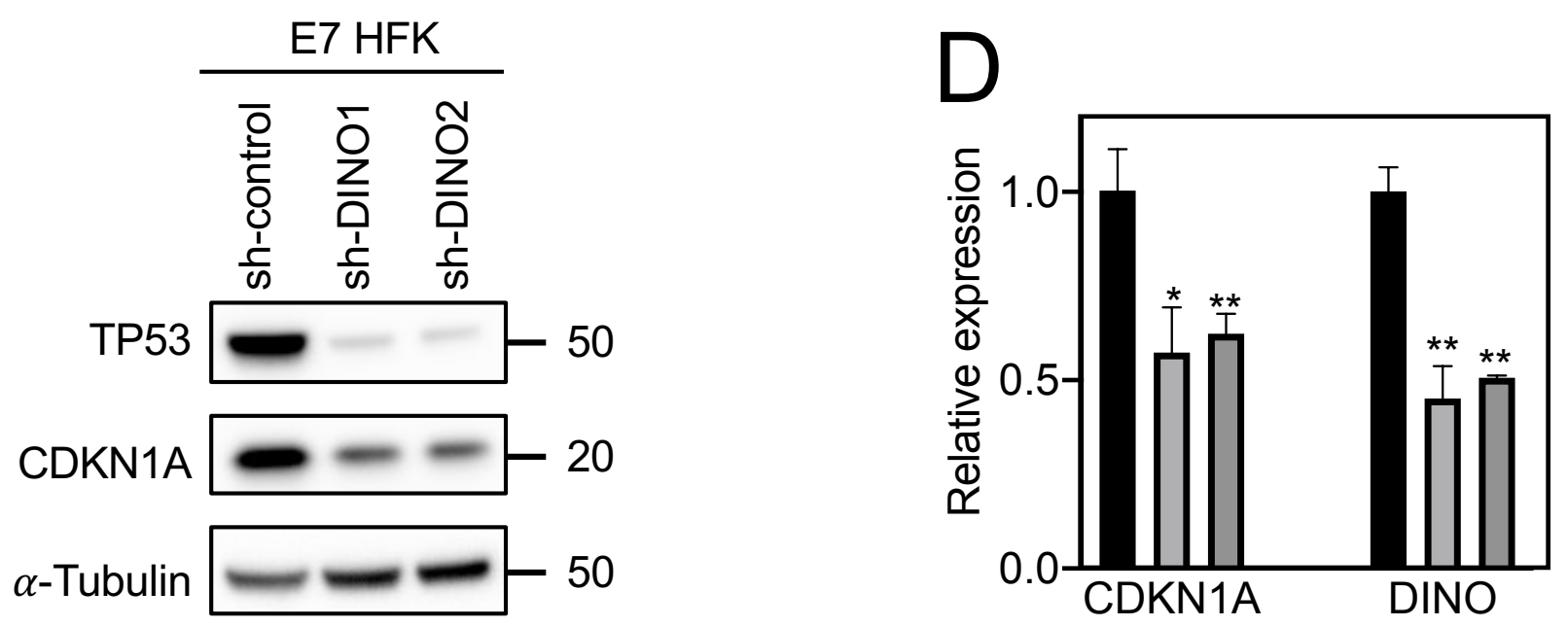

sh-cont

sh-DIN

sh-DIN

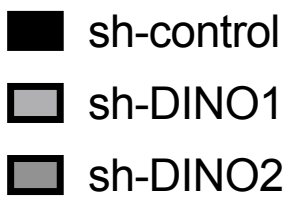

sh-control

$\square$ sh-DINO2 


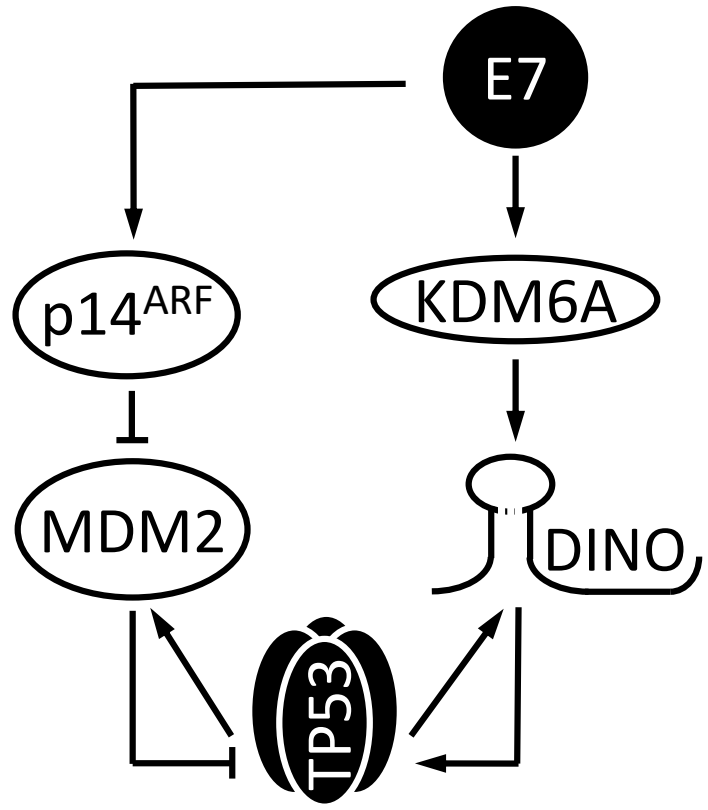

